\title{
Association of Changes in Acute Gastrointestinal Injury Grade with Prognosis in Critically III Patients: A Prospective, Single-Center, Observational Study
}

This article was published in the following Dove Press journal:

Journal of Multidisciplinary Healthcare

\section{Ming Zhongl,* \\ Wen $\mathrm{Xu}^{\mathrm{l}, *}$ \\ Yuzhen Qiu' \\ Lei Li' \\ Hongping $\mathrm{Qu}^{\prime}$ \\ Erzhen Chen ${ }^{2}$}

'Department of Critical Care Medicine, Ruijin Hospital, Shanghai Jiao Tong University School of Medicine, Shanghai, 200025, People's Republic of China; ${ }^{2}$ Department of Emergency, Ruijin Hospital, Shanghai Jiao Tong University School of Medicine, Shanghai, 200025,

People's Republic of China

*These authors contributed equally to this work
Correspondence: Hongping Qu Department of Critical Care Medicine, Ruijin Hospital, Shanghai Jiao Tong University School of Medicine, No. 197. Ruijin No.2 Road, Shanghai 200025, People's Republic of China

Email hongpingqu04I2@hotmail.com

Erzhen Chen

Department of Emergency, Ruijin Hospital, Shanghai Jiao Tong University

School of Medicine, Shanghai 200025,

People's Republic of China

Email rjchenerzhen@I63.com
Purpose: To investigate the association between the change of acute gastrointestinal injury (AGI) grade and the outcome in critically ill patients.

Methods: This was a prospectively observational study. All patients admitted in the ICU from October 2013 to June 2015, with the duration of ICU $>72 \mathrm{~h}$ and age $>18$ years, were enrolled in this study. The AGI grade and gastrointestinal symptoms were evaluated during ICU stay following the 2012 ESICM recommendation. The ICU mortality, duration of ICU stay, mechanical ventilation (MV) use, vasoactive drug use, and continuous renal replacement therapy of patients were recorded accordingly.

Results: A total of 320 patients were included, and 265 of them were diagnosed with AGI. The overall ICU mortality was $11.88 \%$, while it was $13.58 \%$ in patients with AGI. In logistic regression analyses, the decreasing trend of AGI grade was identified as a protective factor for ICU death (odds ratio (OR), 0.484; 95\% confidence interval (CI), 0.26-0.90), while the max AGI grade was a risk factor (OR, 3.464; 95\% CI, 2.71-8.47) for ICU death.

Conclusion: The changes of AGI grades in critically ill patients were associated with their clinical outcomes. The ICU-acquired AGI patients associated with longer ICU stay days.

Keywords: acute gastrointestinal injury grade, ICU stay, critically ill patient, patient outcome, ICU death

\section{Introduction}

Gastrointestinal dysfunction has been considered as an important clinical concern in the critically ill population. ${ }^{1}$ In 2012, the European Society of intensive care medicine (ESICM) released their recommendation on terminology, definitions, and management of gastrointestinal (GI) function in patients in intensive care units (ICU). ${ }^{2}$ In this recommendation, the concept of acute gastrointestinal injury (AGI) was proposed as a malfunction of GI in critically ill patients due to their acute illness, and a four-grade severity system was approached accordingly. One observation study has been reported to modify the AGI grades to two simple ones, and this proposed proposal was quite different from the original four-grade system that is derived from ESICM recommendation and thus may change the validity of the AGI grade system to some extent. ${ }^{3}$

Recent evidences have revealed that the degree of organ dysfunction can be positively associated with the prognosis of patients, while it might also be alleviated when the treatment is applied properly. ${ }^{3-5}$ Therefore, the alteration of organ function may serve as 
an important indicator to guide the clinicians to justify the treatment in time, and eventually improve the clinical outcomes in patients with organ dysfunction. After the ESICM recommendation was released, several observational studies have been performed and demonstrated that AGI grade of patients at ICU admitting was positively associated with ICU severity scores, such as Acute Physiology and Chronic Health Enquiry Evaluation II (APACHE II) score, and therefore can act as a predictor for the outcome of critically ill patients. ${ }^{6-8}$ However, whether GI function grade at ICU admitting can be a good predictor for the ICU patients' outcome remains controversial. $^{3}$ In this study, the association between the changes of AGI grade and the outcomes in critically ill patients was systematically investigated as to verify the effects of AGI grades on ICU patients' outcomes, as well as provide the evidence for clinicians to improve the outcomes of ICU patients with AGI.

\section{Patients and Methods}

\section{Patient Eligibility}

This is an observational cohort study, and it was conducted in a 12-bed surgical ICU of a teaching hospital at the Department of Critical Care Medicine, RuiJin Hospital Affiliated to Shanghai Jiao Tong University School of Medicine, Shanghai, China. All patients admitted from October 2013 to June 2015 were enrolled in this study. The inclusion criteria were: 1) Duration of ICU stay $>72 \mathrm{~h}$, and 2) age $>18$ years. This study was approved by the Ethical Committee of the Ruijin Hospital affiliated to Shanghai Jiao Tong University and conducted in accordance with the Declaration of Helsinki. Patients or their legal representatives were verbally informed about the use of their data for this study, and the consents were signed accordingly.

\section{Data Collection}

All the attending doctors in the department were trained to diagnose the patients with AGI following the 2012 ESICM recommendation. AGI grade and gastrointestinal symptoms were evaluated every day during ICU stay. The other clinical data collected from electrical medication records, including demographic data, APACHE II score, duration of ICU stay, mechanical ventilation (MV) usage, vasopressor usage, continuous renal replacement therapy (CRRT) usage, ICU mortality, were recorded. The primary endpoint was ICU mortality. No specific protocols or recommendations for AGI management were imposed. The patient was treated in a standard procedure in the ICU. All the data were collected until ICU discharge.

\section{AGI Diagnosis}

The gastrointestinal symptoms were defined as follows: Intraabdominal hypertension (IAH), intra-abdominal pressure (IAP) was found to be $12 \mathrm{mmHg}$ or higher, confirmed by at least two measurements; High gastric residual volume, a single gastric residual volume exceeded $200 \mathrm{~mL}$; Diarrhea, three or more loose or liquid stools per day, or a stool more than $250 \mathrm{~g} /$ day; Gastrointestinal bleeding, any bleeding into the GI tract lumen, confirmed by the macroscopic presence of blood in vomited fluids, gastric aspirate, or stool; Paralysis of the lower GI tract, the inability of the bowel to pass stool due to impaired peristalsis; Abnormal bowel sounds, absent peristalsis-no bowel sounds were heard at cautious auscultation; Hyperperistalsis, excessive bowel sounds were heard on auscultation; Bowel dilatation, colonic diameter exceeded $6 \mathrm{~cm}$ (higher than $9 \mathrm{~cm}$ for caecum), or small bowel diameter exceeded $3 \mathrm{~cm}$, diagnosed either on plain abdominal X-ray or computed tomography (CT) scan.

The AGI grades were defined following the 2012 ESICM recommendations as follows: ${ }^{2}$ AGI I grade, the function of the GI tract was partially impaired, GI symptom was temporary and self-limiting. Clinically, it is common in nausea and vomiting after abdominal surgery, loss of bowel sounds, and diminished bowel motility in the early stages of shock; AGI II grade, the general condition of GI dysfunction required therapeutic interventions and was improved after that. The GI tract was not able to perform digestion and absorption adequately to satisfy the nutrient and fluid requirements of the body. There were no changes in the general condition of the patient related to GI problems. Clinical manifestations are gastroparesis with massive gastric retention or reflux, lower gastrointestinal paralysis, diarrhea, IAH grade I (IAP $12 \sim 15 \mathrm{mmHg}$ ), visible bleeding in gastric contents or feces, and food intolerance; AGI III grade, the general condition was not improving despite therapeutic interventions with the deterioration of another organ. Clinical manifestations are massive gastric retention, persistent gastrointestinal paralysis, bowel dilatation, progression of intraabdominal hypertension to grade II (IAP 16 to $20 \mathrm{mmHg}$ ), and decreased abdominal perfusion pressure $(<60 \mathrm{mmHg})$; AGI IV grade, AGI had progressed to become directly and immediately life-threatening, with worsening of Multi-organ Dysfunction Syndrome (MODS), such as intestinal ischemic necrosis, gastrointestinal bleeding leading to hemorrhagic shock, Ogilvie's syndrome (acute colonic pseudoobstruction), abdominal compartment syndrome (ACS) 
Table I Patients' Characteristics

\begin{tabular}{|c|c|c|c|}
\hline & Total & With AGI & Without AGI \\
\hline No. of patients, n (I00\%) & $320(100 \%)$ & $265(82.81 \%)$ & $55(17.29 \%)$ \\
\hline Male, n (\%) & $199(62.19 \%)$ & $166(62.64 \%)$ & $33(60 \%)$ \\
\hline Age, years \pm SD & $63.55 \pm 16.39$ & $63.61 \pm 15.95$ & $63.38 \pm 18.63$ \\
\hline APACHE II score, point (IQR) & $15.00(10.00-20.00)$ & $15.00(11.50-21.00)$ & $10(4-16)$ \\
\hline ICU stay, days (IQR) & $10.00(4.00-21.00)$ & $12.00(6.00-23.00)$ & $5(I-8)$ \\
\hline MV, n (\%) & $138(43.13 \%)$ & $126(47.55 \%)$ & $12(21.81 \%)$ \\
\hline Vasoactive agents, n (\%) & II 2 (35.00\%) & $108(40.75 \%)$ & $4(7.27 \%)$ \\
\hline CRRT, n (\%) & $41(12.81 \%)$ & 40 (I5.09\%) & $\mathrm{I}(\mathrm{I} .18 \%)$ \\
\hline ICU death, n (\%) & $38(11.88 \%)$ & $36(13.58 \%)$ & $2(3.63 \%)$ \\
\hline \multicolumn{4}{|l|}{ Admitted diagnosis, $n(\%)$} \\
\hline Tumor & 137 (42.81\%) & $137(51.7 \%)$ & 0 \\
\hline Pancreatitis & $60(18.75 \%)$ & $60(22.64 \%)$ & 0 \\
\hline Perforation or ileus & $13(4.06 \%)$ & $13(4.91 \%)$ & 0 \\
\hline Bile tract infection & II (3.44\%) & II (4.15\%) & 0 \\
\hline Urology & II (3.44\%) & $4(1.51 \%)$ & $7(12.7 \%)$ \\
\hline Trauma & $4(1.25 \%)$ & $2(0.75 \%)$ & $2(3.63 \%)$ \\
\hline Other & $74(23.13 \%)$ & $38(14.38 \%)$ & $36(65.45 \%)$ \\
\hline
\end{tabular}

requiring aggressive decompression. ICU-acquired AGI, the patient diagnosed AGI after $72 \mathrm{~h}$ in ICU.

\section{Statistical Analysis}

Statistical Package for the Social Sciences Version 16.0 (SPSS 16.0, SPSS Inc., IL, USA) software was used for the statistical analysis. Data were presented as mean \pm standard deviation (SD) if normally distributed, otherwise presented as median \pm interquartile range (IQR). For comparisons between groups, Student's $t$-test (normal distribution) and Mann-Whitney $U$-test (non-Gaussian distribution) were used for continuous variables and Chi-square test was performed for categorical variables. The Cochran-Armitage Trend Test was conducted to test the trend in categorical variables. Spearman correlation was used in continuous variables. The forward likelihood ratio was used to build a logistic regression model to identify the risk factors for ICU mortality. We set factors as follows: age, APACHE II score, the max AGI grade, AGI grade day 1 , AGI grade day 3, AGI grade day 7, AGI grade decreasing trend from day 1 to day 3 (Upgrade, no change, and downgrade patient cohorts), AGI grade decreasing trend from day 1 to day 7 (Upgrade, no change, and downgrade patient cohorts). AGI grade decreasing trend is used as a categorical variable. If AGI grade increases, it is marked as upgrade (assigned value 1); if AGI grade does not increase, it is marked as downgrade or no change (assigned value 0 ). The likelihood ratio (forward) was used to construct the model from day 1 to day 7. An OR equal to 1 indicates that the factor does not play a role in the occurrence of the disease; an OR more than 1 indicates that the factor is a risk factor; and an OR less than 1 indicates that the factor is a protective factor. $\mathrm{P}<0.05$ was considered statistically significant differences. Calibration progress: Re-evaluation by at least two physicians familiar with the AGI evaluation process.

\section{Results}

\section{Patients' Characteristics and the Incidence of AGI}

A total of 338 patients were recruited from October 2013 to June 2015, while 18 of them were excluded from the study and 320 patients were eventually eligible in this study. There were 265 patients $(82.81 \%)$ who were diagnosed with AGI. The included patients' characteristics were presented in Table 1. The average age of patients on admission was $63.55 \pm$ 16.39 years, and the median APACHE II score was 15.00. The ICU mortality of the whole study population was $11.88 \%$, while it was $13.58 \%$ in patients with AGI $(\mathrm{p}=0.728)$. The GI symptoms, such as the abnormal bowel sounds, paralysis of lower GI tract, high gastric residual volume, diarrhea, GI bleeding, vomiting, and bowel dilatation, were sorted by the max AGI grades and listed in Table 2. 
Table 2 Gastrointestinal Symptoms with Different AGI Grades

\begin{tabular}{|l|l|l|l|l|}
\hline & AGI I & AGI II & AGI III & AGI IV \\
\hline Abnormal bowel sounds, n (\%) & $78(67.83)$ & $46(38.33 \%)$ & $23(34.85 \%)$ & II (39.29\%) \\
Paralysis of lower Gl tract, n (\%) & $24(20.87 \%)$ & 3 I (25.83\%) & $17(25.76 \%)$ & $7(25.00 \%)$ \\
High gastric residual volume n (\%) & $3(2.61 \%)$ & $13(10.83 \%)$ & $5(7.58 \%)$ & $2(7.14 \%)$ \\
Diarrhea, n (\%) & $3(2.61 \%)$ & $9(7.50 \%)$ & $6(9.09 \%)$ & I (3.57\%) \\
Gl bleeding, n (\%) & $3(2.61 \%)$ & $8(6.67 \%)$ & $5(7.58 \%)$ & $3(10.71 \%)$ \\
Vomiting (emesis), n (\%) & $3(2.61 \%)$ & $9(7.50 \%)$ & $5(7.58 \%)$ & I (3.57\%) \\
Bowel dilatation, n (\%) & I (0.87\%) & $4(3.33 \%)$ & $5(7.58 \%)$ & $3(10.71 \%)$ \\
\hline
\end{tabular}

Abbreviations: AGI, acute gastrointestinal injury; n, number; $\mathrm{Gl}$, gastrointestinal.

Table 3 ICU-Acquired AGI vs Non-ICU-Acquired AGI

\begin{tabular}{|l|l|l|l|}
\hline & ICU-Acquired AGI & Non-ICU-Acquired AGI \\
\hline No. of patients, n (\%) & 45 & 220 & \\
APACHE II score, IQR & $17.00(13.00-23.50)$ & $15.00(11.00-21.00)$ & $P=0.060^{*}$ \\
ICU stay days, IQR & $19.00(10.00-30.00)$ & $11.50(5.25-21.00)$ & $P=0.004^{*}$ \\
MV (\%) & $28(62.22 \%)$ & $98(44.55 \%)$ & $P=0.03 I^{* *}$ \\
Vasoactive agents (\%) & $20(44.44 \%)$ & $88(40.00 \%)$ & $\mathrm{P}=0.580^{* *}$ \\
CRRT (\%) & $7(15.56 \%)$ & $33(15.00 \%)$ & $\mathrm{P}=0.924^{* *}$ \\
ICU death (\%) & $6(13.33 \%)$ & $30(13.64 \%)$ & $\mathrm{P}=0.957^{* *}$ \\
\hline
\end{tabular}

Notes: *Wilcoxon test; **Chi-square test.

Abbreviations: ICU, intensive care unit; AGI, acute gastrointestinal injury; n, number; APACHE II, Acute Physiology and Chronic Health Enquiry; IQR, interquartile range; $\mathrm{MV}$, mechanical ventilation; CRRT, continuous renal replacement therapy.

\section{ICU-Acquired AGI Patients}

ICU-acquired AGI was defined when the patient was diagnosed with AGI after $24 \mathrm{~h}$ in ICU. According to this standard, there were 45 patients (16.98\%) with ICU-acquired AGI, among which 19 patients $(42.22 \%)$ were first documented AGI within 3 days in ICU, 17 patients (37.78\%) were documented between the 3rd to the 7th days, 6 patients (13.33\%) were within the 2nd week, and only $3(6.67 \%)$ were firstly diagnosed with AGI after 2 weeks from the admission. Compared with non-ICU-acquired AGI, these patients had a significantly longer ICU stay days (19.00 (10.00-30.00) vs 11.50 (5.25-21.00), $\mathrm{p}=0.004)$, and more MV usages (62.22\% vs $44.55 \%, \mathrm{p}=0.031)$. There were no significant differences in the ICU mortalities, the usage of vasoactive drugs, and the usage of CRRT between the ICU-acquired AGI and non-ICUacquired AGI groups (Table 3).

\section{The Association of AGI Grades and Outcome for ICU Patients}

Next, we explored the association of AGI grades with the outcomes in ICU patients. The proportion of each AGI grade is shown in Table 4. It was demonstrated that an increasing mortality trend with AGI grade on the first ICU day and with max AGI grade. Meanwhile, it was presented the same trend in the case of receiving mechanical ventilation, vasopressor usage, renal replacement therapy (Table 4).

There were 206 patients who stayed in ICU for more than 7 days. We recorded the change of their AGI grades and analyzed the relationship with their outcomes. Comparing day 1 and day 3, the AGI grades were upgraded in 30 patients (14.56\%), with no change in 158 patients $(76.7 \%)$, and were downgraded in 18 patients (8.74\%) (Table 5). Their ICU mortalities, ICU stay days, hospitalization days, the ratios of CRRT, MV or vasoactive agents' usage between day 1 and day 7 were not statistically different (Table 5).

When compared with day 1 and day 7, the AGI grade was upgraded in 41 patients (19.90\%), with no change in 96 patients $(46.60 \%)$, while it was downgraded in 69 patients (33.50\%). Meanwhile, the ICU mortalities showed a significantly decreasing trend, with $24.39 \%, 14.58 \%$, and $5.80 \%$ in Upgrade, No change, and Downgrade cohorts of patients, respectively ( $\mathrm{p}=0.006$, Table 6 ). Additionally, the ratios of CRRT were significantly reduced following the downgraded AGI, with $29.27 \%, 18.75 \%$, and $8.70 \%$ in Upgrade, No change, and Downgrade groups, respectively $(\mathrm{p}=0.005$, Table 6). There were no significant differences in ICU stay days, hospitalization days, MV or vasoactive agents' usages from day 1 to day 7 in the three patient cohorts (Table 6). 


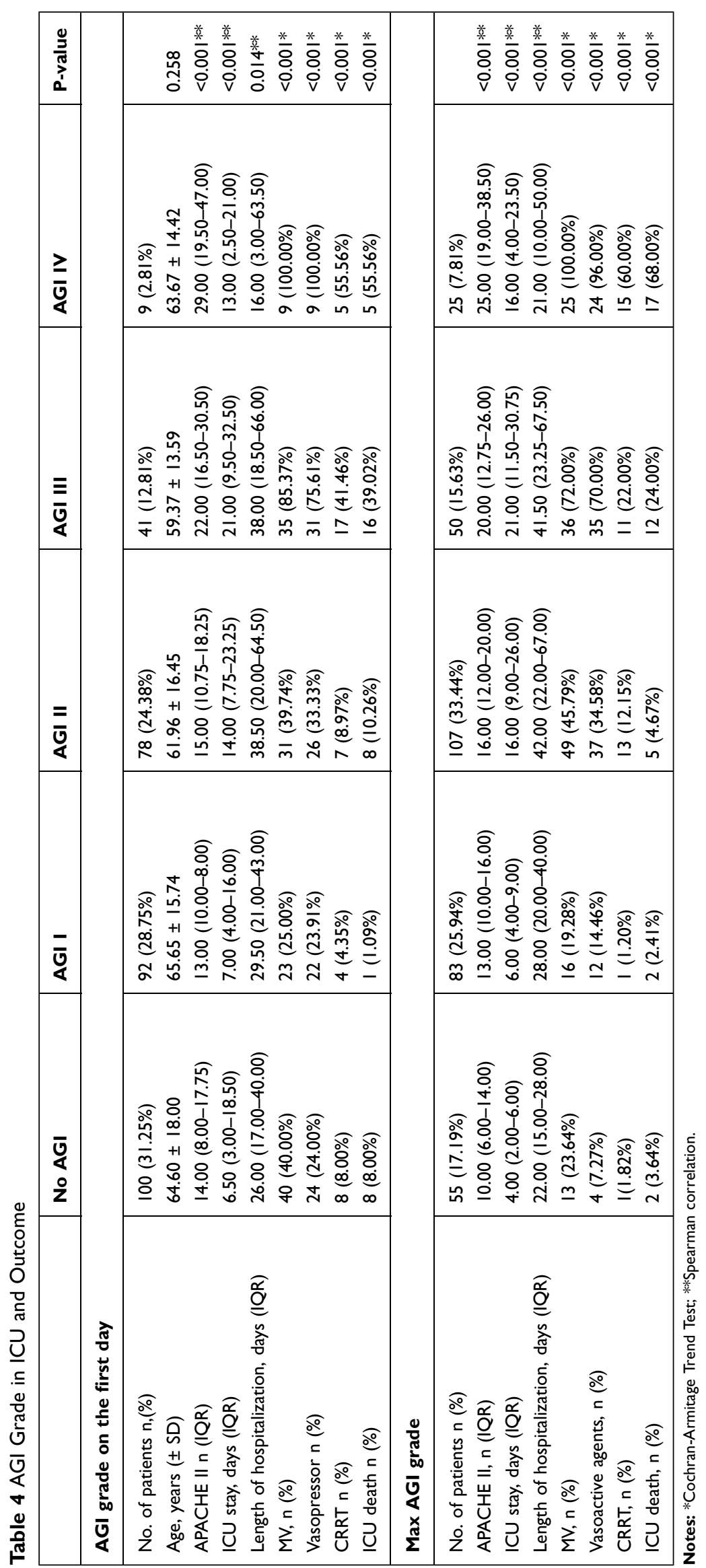


Table 5 Change in AGI Grade Day I vs Day 3

\begin{tabular}{|l|l|l|l|l|}
\hline & Upgraded & No Change & Downgraded & P-value \\
\hline No. of patients, n (\%) & $30(14.56 \%)$ & $158(76.70 \%)$ & $18(8.74 \%)$ & \\
ICU stay, days (IQR) & $17.00(10.75-25.25)$ & $16.50(10.00-25.00)$ & $23.50(12.75-45.50)$ & $0.236 * *$ \\
Length of hospitalization, days (IQR) & $35.50(23.25-61.50)$ & $37.50(23.00-67.50)$ & $56.50(28.25-77.25)$ & $0.328^{* *}$ \\
MV (\%) & $21(70.00 \%)$ & $85(53.80 \%)$ & $11(61.11 \%)$ & $0.25 *$ \\
Vasoactive agents (\%) & $19(63.33 \%)$ & $67(42.41 \%)$ & $10(55.56 \%)$ & $0.079 *$ \\
CRRT (\%) & $7(23.33 \%)$ & $28(17.72 \%)$ & $1(5.56 \%)$ & $0.288^{*}$ \\
ICU death (\%) & $4(13.33 \%)$ & $22(13.92 \%)$ & $2(I 1.11 \%)$ & $1.0 *$ \\
\hline
\end{tabular}

Notes: *Cochran-Armitage Trend Test; **Spearman correlation.

Abbreviations: $\mathrm{AGI}$, acute gastrointestinal injury; $\mathrm{n}$, number; ICU, intensive care unit; IQR, interquartile range; MV, mechanical ventilation; CRRT, continuous renal replacement therapy.

Table 6 Change in AGI Grade Day I vs Day 7

\begin{tabular}{|l|l|l|l|l|}
\hline & Upgrade & No Change & Downgrade & P-value \\
\hline n (\%) & $4 I(19.90 \%)$ & $96(46.60 \%)$ & $69(33.50 \%)$ & $13.00(8.00-25.50)$ \\
ICU stay, days (IQR) & $20.00(12.50-31.50)$ & $17.00(11.00-24.75)$ & & $0.124 * *$ \\
Length of hospitalization, days (IQR) & $32.00(20.50-62.00)$ & $37.00(23.00-64.00)$ & $44.00(23.00-76.00)$ & $0.393 * *$ \\
MV, n (\%) & $27(65.85 \%)$ & $57(59.38 \%)$ & $33(47.83 \%)$ & $0.053 *$ \\
Vasoactive agents, n (\%) & $23(56.10 \%)$ & $44(45.83 \%)$ & $29(42.03 \%)$ & $0.17 I^{*}$ \\
CRRT, n (\%) & $12(29.27 \%)$ & $18(18.75 \%)$ & $6(8.70 \%)$ & $0.005 *$ \\
ICU death, n (\%) & $10(24.39 \%)$ & $14(14.58 \%)$ & $4(5.80 \%)$ & $0.006 *$ \\
\hline
\end{tabular}

Notes: *Cochran-Armitage Trend Test; **Spearman correlation.

Abbreviations: AGI, acute gastrointestinal injury; n, number; ICU, intensive care unit; IQR, interquartile range; MV, mechanical ventilation; CRRT, continuous renal replacement therapy.

\section{Logistic Regression Analysis to Identify Risk Factors for ICU Death}

In logistic regression analysis, the result showed the decreasing trend of AGI grade was identified as a protective factor for ICU death (odds ratio (OR), 0.484; 95\% confidence interval (CI), 0.26-0.90), while the max AGI grade was a risk factor (OR, 3.464; 95\% CI, 2.71-8.47) for ICU death (Table 7).

\section{Discussion}

GI dysfunction is commonly diagnosed in critically ill population. ${ }^{9}$ It has been reported that the prevalence of GI dysfunction is about $40 \%$ in critically ill patients. ${ }^{4}$ In

Table 7 Logistic Regression on Risk Factors for ICU Survival from Day I to Day 7

\begin{tabular}{|l|l|l|l|}
\hline & OR & P-value & $\mathbf{9 5 . 0 \%} \mathbf{~ C l}$ \\
\hline AGl grade decreasing trend & 0.484 & 0.02 & $0.26-0.90$ \\
Max AGl grade & 3.464 & 0.00 & $2.71-8.47$ \\
\hline
\end{tabular}

Abbreviations: ICU, intensive care unit; OR, odds ratio; $\mathrm{Cl}$, confidence interval; AGI, gastrointestinal injury; APACHE II, Acute Physiology and Chronic Health Enquiry. this study, we found more than $80 \%$ of ICU patients could develop AGI during their stay in ICU. It was indicated that the levels of max AGI grades were in correlation with the need for mechanical ventilation, vasopressor agents, renal replacement therapy, and so on. The results showed that the higher the max AGI grade the patients were diagnosed with, the worse outcome they may obtain. Meanwhile, this study also demonstrated that the mortality of patients in ICU was significantly associated with AGI grade. These findings were consistent with other studies. ${ }^{3,5,10,11}$ Collectively, this study indicated that the GI function is of utmost importance in surgical patients, and meanwhile maintaining a good GI function would greatly improve the outcome of patients.

GI function may dynamically change as the overall condition of patient changes during the stay of ICU. In this study, we introduced the conception of "ICU-acquired AGI", which was defined as a newly discovered AGI in ICU despite how it was acquired by the patients. This concept was based on the idea that GI failure can be a consequence of other organ failure progressing. If the patients become worse in ICU, they may develop GI injury 
even though they may not manifest GI injury when they were admitted. In this study, we showed that patients with ICU-acquired AGI stayed longer than those with non-ICUacquired AGI, and it was more likely for ICU-acquired AGI patients to require for $\mathrm{MV}$, which indicated that ICUacquired AGI can be an expression of the worse condition of ICU patients. However, the mortality in ICU-acquired AGI and non-ICU-acquired AGI groups was not significantly different, which was not consistent with a previous study. ${ }^{11}$ Future study with large size of samples will be required to further confirm the current result.

GI symptoms are important clinical detection index for GI function, but usually, they are difficult to be diagnosed for the patients by the clinicians. ${ }^{12}$ Take feeding intolerance as an example, it usually took up to $72 \mathrm{~h}$ to clinically confirm the GI symptom in patients. Although close observation on GI function of ICU patients is essential, it is difficult to select the best way in a patient or a unit. In addition, the identification of GI dysfunction may be influenced by many other factors, such as the unstable hemodynamic state of the patient could prevent clinicians from evaluating the tolerance, as well as the different feeding protocol among different units. ${ }^{13}$ As GI dysfunction can be a sequence of deterioration of another organ, and medical practice, such as MV, has been well known to be a cause of GI dysfunction, ${ }^{14}$ the patients may need several days to treat the primary organ dysfunction (such as shock, respiratory failure, or renal failure), before which the consequent GI dysfunction is difficult to be improved. It may be insufficient for the clinicians to observe patients' GI function in those who stay less than 7 days. Thus, only patients stay no less than 7 days were analyzed to check the relation of GI symptom changing and outcomes. Therefore, a more rapid and reliable way to evaluate the GI function is still desirable in the clinical settings.

During the study, it was challenging to obtain an objective grade of AGI for each patient. Hence, we applied a calibration progress to define each of the AGI grade. This helped us to better understand the ESICM recommendation, as well as diagnose and deal with these AGI patients. In this study, we demonstrated that the change of AGI grade on the 7th ICU day might be associated with ICU patients' outcomes. The logistic regression has confirmed that the ICU mortality was in positive correlation with the AGI grade in critically ill patients. This indicated that when the GI injury in a critically ill patient can be controlled within 7 days' treatment, a better outcome can be expected. Meanwhile, it was reported that the GI function improvement might also be a result of alleviated primary organ dysfunction or disease. ${ }^{15}$ Therefore, dynamic evaluation of the AGI grade of critically ill patients can serve as a good tool to predict patients' outcomes. However, in the current study, it did not show the same result in patients with a worse AGI grade on the 3rd in the ICU. This may be explained that in most of the cases, it usually takes $24 \sim 48 \mathrm{~h}$ to initialize the enteral nutrition for the patients in the ICU. On the other hand, the GI function is mainly evaluated along with other nonspecific symptoms by the clinician, and it may not be accurately determined if the GI function is improved by the treatment or not within the first 3 days. ${ }^{16}$ Importantly, the protocols of bedside ultrasonography evaluation have been developed for GI function detection, and are reported to be a real-time and simple approach that can be applied in the ICU. Though ultrasonography may improve the accuracy for detection of the GI function, the interpretation and operation of ultrasonography are still highly operator-dependent. ${ }^{17}$ Further study with ultrasonography evaluation of GI function in patients in the ICU is needed to confirm our current findings.

There are still some limitations to our study. Firstly, this study was conducted in a single center, and patients were mostly admitted after the surgery. Therefore, the result may be difficult to represent the general population of patients in the ICU. Secondly, as an observational study, it showed the association but not the causality between the AGI and outcome of the patients. Thirdly, the study was not involved any biomarker (such as citrulline, intestinal fat acid-binding protein, intestinal alkaline phosphatase, etc.) for GI function in patients. Additionally, it is reported that the intestinal microbiome also plays important roles in GI function, but the test is costly and difficult to operate clinically. Further investigations are warranted to verify the correlation between AGI grade and its biomarkers or microbiome change in critically ill patients.

\section{Conclusion}

This small sample of the pilot study showed the incidence of AGI was high in the study population. The changes in AGI grades were associated with the patients' outcomes, and ICU-acquired AGI was related to longer ICU stay days.

\section{Acknowledgments}

Ming Zhong and Wen $\mathrm{Xu}$ are co-first authors for this study. We also want to thank all the staffs in our department for their support and endeavor for this study. 


\section{Funding}

Shanghai Shenkang Hospital Development Centre of China, Program for Outstanding Medical Academic Leader (SHDC12017116); Science and Technology Commission of Shanghai Municipality (No.18411950900); National Nature Science Foundation of China (81772107); Wu Jieping Medical Foundation (NO. 320.6750.18497); China international medical foundation NO. z-2017-24-1866.

\section{Disclosure}

The authors report no conflicts of interest in this work.

\section{References}

1. Klek S, Forbes A, Gabe S, et al. Management of acute intestinal failure: a position paper from the European Society for Clinical Nutrition and Metabolism (ESPEN) special interest group. Clin Nutr. 2016;35(6):1209-1218. doi:10.1016/j.clnu.2016.04.009

2. Reintam Blaser A, Malbrain ML, Starkopf J, et al. Gastrointestinal function in intensive care patients: terminology, definitions, and management. Recommendations of the ESICM working group on abdominal problems. Intensive Care Med. 2012;38(3):384-394. doi:10.1007/ s00134-011-2459-y

3. Li H, Zhang D, Wang Y, et al. Association between acute gastrointestinal injury (AGI) grading system and disease severity and prognosis in critically ill patients: a multicenter, prospective, observational study in China. J Crit Care. 2016;36:24-28. doi:10.1016/j.jcrc.2016.05.001

4. Zhang D, Li Y, Ding L, et al. Prevalence and outcome of acute gastrointestinal injury in critically ill patients: a systematic review and meta-analysis. Medicine (Baltimore). 2018;97(43):e12970. doi:10.1097/MD.0000000000012970

5. $\mathrm{Hu} \mathrm{B}$, Sun R, Wu A, et al. Severity of acute gastrointestinal injury grade is a predictor of all-cause mortality in critically ill patients: a multicenter, prospective, observational study. Crit Care. 2017;21 (1):188. doi:10.1186/s13054-017-1780-4

6. Lee H, Lim CW, Hong HP, et al. Efficacy of the APACHE II score at ICU discharge in predicting post-ICU mortality and ICU readmission in critically ill surgical patients. Anaesth Intensive Care. 2015;43 (2):175-186. doi:10.1177/0310057X1504300206
7. Nik A, Sheikh Andalibi MS, Ehsaei MR, et al. The efficacy of Glasgow Coma Scale (GCS) Score and Acute Physiology and Chronic Health Evaluation (APACHE) II for predicting hospital mortality of ICU patients with acute traumatic brain injury. Bull Emerg Trauma. 2018;6(2):141-145. doi:10.29252/beat-060208

8. Godinjak A, Iglica A, Rama A, et al. Predictive value of SAPS II and APACHE II scoring systems for patient outcome in a medical intensive care unit. Acta Med Acad. 2016;45(2):97-103. doi:10.5644/ ama2006-124.165

9. Hill TL. Gastrointestinal tract dysfunction with critical illness: clinical assessment and management. Top Companion Anim Med. 2019;35:47-52. doi:10.1053/j.tcam.2019.04.002

10. Ding L, Chen HY, Wang JY, et al. Severity of acute gastrointestinal injury grade is a good predictor of mortality in critically ill patients with acute pancreatitis. World J Gastroenterol. 2020;26(5):514-523. doi:10.3748/wjg.v26.i5.514

11. Zhong $\mathrm{M}, \mathrm{Xu} \mathrm{W}, \mathrm{Li} \mathrm{L}$, et al. Incidence of acute gastrointestinal injury in ICU patients. J Chin Pract Diagn Ther. 2016;30:169-171.

12. Reintam Blaser A, Jakob SM, Starkopf J. Gastrointestinal failure in the ICU. Curr Opin Crit Care. 2016;22(2):128-141. doi:10.1097/ MCC.0000000000000286

13. Boullata JI, Carrera AL, Harvey L, et al. ASPEN safe practices for enteral nutrition therapy. JPEN J Parenter Enteral Nutr. 2017;41 (1):15-103. doi:10.1177/0148607116673053

14. Gungabissoon U, Hacquoil K, Bains C, et al. Prevalence, risk factors, clinical consequences, and treatment of enteral feed intolerance during critical illness. JPEN J Parenter Enteral Nutr. 2015;39 (4):441-448. doi:10.1177/0148607114526450

15. Heyland DK, Tougas G, King D, et al. Impaired gastric emptying in mechanically ventilated, critically ill patients. Intensive Care Med. 1996;22(12):1339-1344. doi:10.1007/BF01709548

16. Perez-Calatayud AA, Carrillo-Esper R, Anica-Malagon ED, et al. Point-of-care gastrointestinal and urinary tract sonography in daily evaluation of gastrointestinal dysfunction in critically ill patients (GUTS Protocol). Anaesthesiol Intensive Ther. 2018;50(1):40-48. doi:10.5603/AIT.a2017.0073

17. Piton G, Capellier G. Biomarkers of gut barrier failure in the ICU. Curr Opin Crit Care. 2016;22(2):152-160. doi:10.1097/ MCC.0000000000000283
Journal of Multidisciplinary Healthcare

\section{Publish your work in this journal}

The Journal of Multidisciplinary Healthcare is an international, peerreviewed open-access journal that aims to represent and publish research in healthcare areas delivered by practitioners of different disciplines. This includes studies and reviews conducted by multidisciplinary teams as well as research which evaluates the results or conduct of such teams or healthcare processes in general. The journal covers a very wide range of areas and welcomes submissions from practitioners at all levels, from all over the world. The manuscript management system is completely online and includes a very quick and fair peer-review system. Visit http://www.dovepress.com/testimonials. php to read real quotes from published authors. 